\title{
Short Term Evaluation Biological Bone Reconstruction for Tibia Distal Osteosarcoma
}

\author{
Misbah Huddin ${ }^{1 *}$, Mujaddid Idulhaq ${ }^{2}$ \\ ${ }^{1}$ Orthopaedic and Traumatologi Division, Sebelas Maret University, Prof. Dr. R. Soeharso Orthopaedic Hospital, Surakarta, \\ Central Java, Indonesia; ${ }^{2}$ Orthopedic Musculoskeletal Tumor Division, Sebelas Maret University, Prof. Dr. R. Soeharso \\ Orthopaedic Hospital, Surakarta, Central Java, Indonesia
}

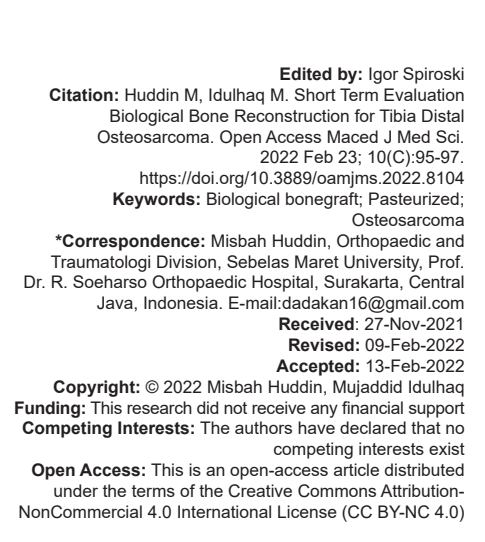

\section{Abstract}

INTRODUCTION: Limb salvage in children with primary malignant bone tumors in lower extremity is challenging, with considerations such as shortening, bone and joint remodeling and high functional demands of active children. We adopted a biological approach by preserving the patient native joint, with bony defects bridged by autologous tibial biological grafts

PRESENTATION OF CASE: A 16-year-old female with pain in the left lower leg and was diagnosed with a tibia distal osteosarcoma. The patient was perform preoperative chemotherapy and had a good result. This was assessed clinically by a decrease in the size of the swelling and pain from the tumor, and radiological. Then, she underwent wide excision surgery, we decided to perform biological reconstruction with pasteurized technic, and evaluation after 3 month (union, early weight-bearing and rehabilitation). We found that biological reconstruction using pasteurized technic with autologous tibial biological grafts is a viable option in children with high-grade osteosarcoma in the lower extremity. It provides immediate stability, allows early weight-bearing and early rehabilitation. Time to full weight bearing is shorter than prosthetic replacement. The use of autologous tibial biological grafts has lower cost, no rejection because the patient's own tissue is used, exact anatomical matching and no donor morbidity.

CONCLUSION: We have observed this technique of limb salvage offers satisfactory limb function with short-term follow-up.

\section{Introduction}

Osteosarcoma is the most common primary malignant bone tumor in children. Previously, the prognosis of osteosarcoma is very poor with $<20 \%$ of 5 -year survival rate even after aggressive amputation [1]. In recent years, the use of neoadjuvant chemotherapy has increased the 5-year survival rate of osteosarcoma patients to $60-80 \%$, and advances in surgical techniques have enabled limb salvage in over $80 \%$ of the patients [1]. Epiphysis and metaphysis of the long bones are the most frequent site of osteosarcoma, which is often required resection of the joints [1].

Limb salvage is now widely accepted as an alternative to amputation to achieve local control of the tumor and the feasibility of limb salvage is depended on tumor biology, anatomical site, age, and socioeconomic factors [2]. Limb salvage has changed from being secondary to standard practice in the management of primary malignant bone tumors. Limb salvage surgery represents a challenge in skeletally immature patients whom further growth is anticipated. The selected treatment method should address the current bone defect and the expected leg length discrepancy at mature age [3]. Surgeons have several choices for the reconstruction of large bone defects after tumor resection, for example, endoprotheses, allografts, vascularized fibular grafts, composite arthroplasty, distraction osteogenesis, and biological reconstruction. Biological reconstruction by reusing the resected tumorbearing bone is steadily increasing, through the use of extracorporeal irradiation, autoclaving, pasteurization, and freezing [3].

The common advantage of these techniques is the coincidence of configuration of the bone defects and the reconstructive material so that the reconstructive procedure can be performed easily [4]. Manabe et al., reported that the osteoinductivity and mechanical strength of pasteurized bone were about as similar to those of fresh bone grafts and better than boiled or autoclaved bone, and that heating in over 5 I of water at $60^{\circ} \mathrm{C}$ for about 30 min was sufficient for killing all tumor cells in any massive bone [5]. Based on the results of this experimental study, we have performed reconstruction by pasteurized autogenous bone graft. 


\section{Presentation of Case}

A 16-year-old girl was referred to our clinic with persistent right leg pain and swelling for 3 months joint without cause. From physical examination on left leg region: We found skin intact, no venectation and ulcer, there is mass size $\pm 10 \times 7.5 \times 6 \mathrm{~cm}$, solid, fixed, tenderness $(+)$, temperature $=$ surround skin. Full range of movement (ROM) of knee joint and limited ankle joint due to pain. Radiographic examination indicated a typical periosteal reaction and new bone formation adjacent to the bone at distal tibial (Figure 1).
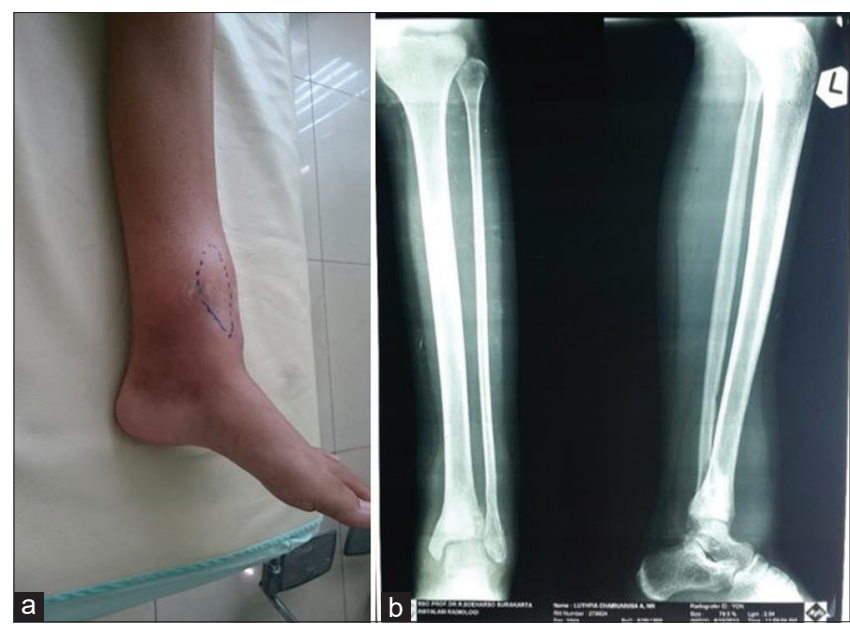

Figure 1: (a) Clinical feature left lower leg, (b) Radiograph left lower limb

A fine needle biopsy of the lesion was performed immediately and the result of its histopathologic diagnostic was osteosarcoma. Whole-body bone scan and computed tomography of the thorax were carried out to search for possible skipped lesions and metastases; none of them were found. Following a magnetic resonance imaging (MRI)

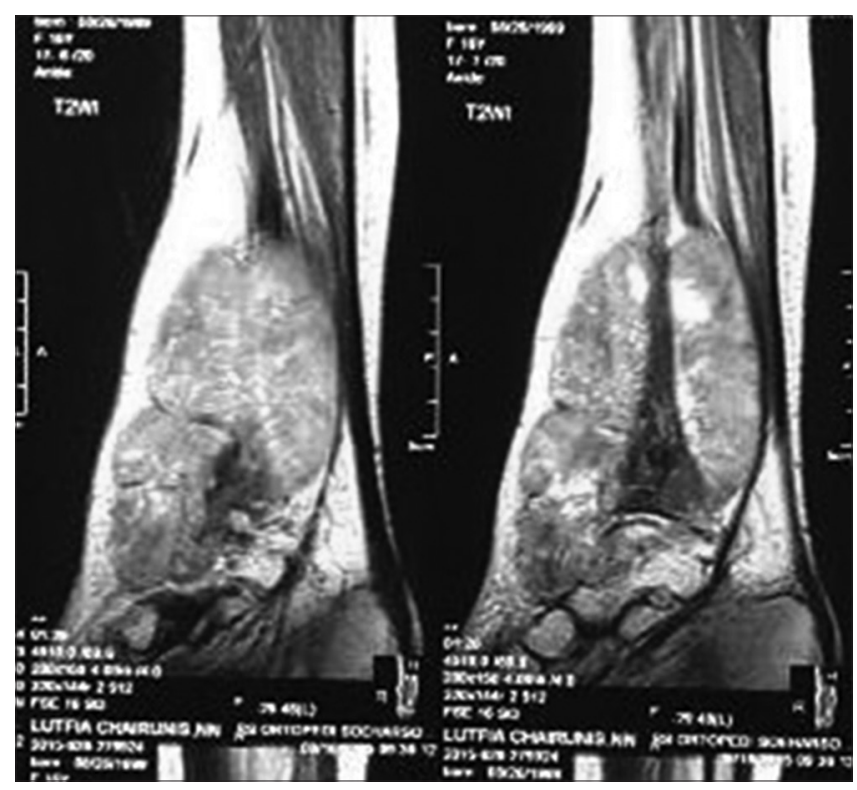

Figure 2: Magnetic resonance imaging axial left lower leg investigation, the tumor was staged as IIB according to the Enneking classification (Figure 2). She underwent seven cycles of neoadjuvant chemotherapy with carboplatin and etoposide. After seven cycles neoadjuvant chemotherapy, we evaluated (restaging), and found good response chemotherapy. Followed by a radiological evaluation including anteroposterior and lateral plain radiograms and MRI, we started to build surgical planning.

The surgical plan was to biologically reconstruct the bone defect, which is would be remained after wide resection of the tumor, using the pasteurized technique with autologous tibial biological autograft (Figure 3). Patients were encouraged post-operative ROM exercises immediately. Touch-down weight bearing using crutches was allowed three months after surgery and weightbearing protection was continued until sufficient callus at the junction between normal was seen radiographically. Full weight-bearing was allowed when solid union was evidenced. Functional evaluation of the patients was performed using the revised 30-point functional classification system established by the International Society of Limb Salvage and the Musculoskeletal Tumor Society (MSTS). The functional score measured six parameters: pain, function, emotional acceptance, use of walking supports, walking ability, and gait. Each parameter is given a value ranging from 0 to 5 , according to specific criteria. The individual scores are added together to obtain an overall functional score, with 30 points as the maximum score for a patient to gain, which is then expressed as normal function. A score of 23 points or greater is considered as an excellent functional result, 15 to 22 points as a good result, 8 to 14 points as a fair result, and $<8$ points defined as a poor result [6].

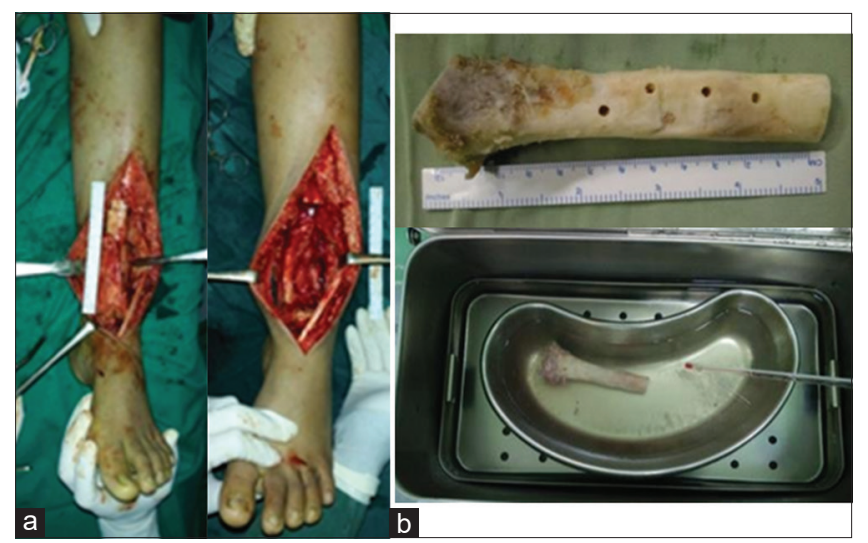

Figure 3: (a) Clinical featrure during operation, (b) Pasteurized autografth

In our patient, we evaluated her condition using MSTS when she came to us for the $1^{\text {st }}$ time and three months after operation had been done. Significant progress was found between $1^{\text {st }}$ and $2^{\text {nd }}$ time scoring of MSTS in the patient. The $1^{\text {st }}$ time scoring resulted in 7 of 30 maximum points while the second one had 18 of 30 maximum points done. This result showed great difference which is very promising for the patient's prognosis. 


\section{Discussion}

There are multiple ways to devitalize remaining tumor cells in the resected bone including boiling, autoclave, pasteurization, liquid nitrogen, and radiation. By heating the bone to $70^{\circ}$ for $30 \mathrm{~min}$, it was shown to adequately devitalize all the tumor cells in the bone. Higher temperature for long duration by autoclaving or boiling will affect the mechanical strength and osteoinductive properties of the bone. This was explained by Urist et al. that higher temperature affects the collagen structure of bone [1]. The stress to failure in compression test was reduced to $55 \%$ and $57 \%$ and modulus of elasticity was reduced to $66 \%$ and $75 \%$ for autoclave and boiling respectively. On the opposite, there is no reduction of mechanical properties after pasteurization [2]

\section{Conclusion}

We have observed this technique of limb salvage offers satisfactory limb function with short-term follow-up.

\section{References}

1. Han G, Wang Y, Bi W, Jia J, Wang W, Xu M, et al. Reconstruction using massive allografts after resection of extremity osteosarcomas the study design: A retrospective cohort study. Int J Surg. 2015;21:108-11. https://doi.org/10.1016/j. ijsu.2015.07.686

PMid:26232712

2. Rougraff BT, Simon MA, Kneisl JS, Greenberg DB, Mankin HJ Limb salvage compared with amputation for osteosarcoma of the distal end of the femur. A long-term oncological, functional, and quality-of-life study. J Bone Joint Surg. 1994;76(5):649-56. https://doi.org/10.2106/00004623-199405000-00004 PMid:8175811

3. Hamed KA, Yamamoto N, Hayashi K, Takeuchi A, Miwa S, Tsuchiya $\mathrm{H}$. Epiphyseal sparing and reconstruction by frozen bone auto-graft after malignant bone tumor resection in children. Hindawi Publ Corporation Sarcoma. 2015;2015:892141.

4. Nishida J, Shimamura T. Methods of reconstruction for bone defect after tumor excision: A review of alternatives. Med Sci Monitor. 2008;14(8):RA107-13. PMid: 18668007

5. Manabe J, Kawaguchi N, Matsumoto S. Pasteurized autogenous bone graft for reconstruction after resection of malignant bone and soft tissue tumors: Imaging features. Semin Musculoskelet Radiol. 2001;5(2):195-201. https://doi. org/10.1055/s-2001-15680 PMid:11500166

6. Enneking WF. Limb Salvage in Musculoskeletal Oncology New York, USA: Churchill-Livingstone; 1987. 